University of Nebraska - Lincoln DigitalCommons@University of Nebraska - Lincoln

\title{
Non-Linear Filtering and Limiting in High Order Methods for Ideal and Non-Ideal MHD
}

Helen C. Yee

NASA Ames Research Center, yee@nas.nasa.gov

Bjorn Sjögreen

Royal Institute of Technology, bjorns@nada.kth.se

Follow this and additional works at: http://digitalcommons.unl.edu/nasapub

Yee, Helen C. and Sjögreen, Bjorn, "Non-Linear Filtering and Limiting in High Order Methods for Ideal and Non-Ideal MHD" (2006). NASA Publications. 266.

http://digitalcommons.unl.edu/nasapub/266

This Article is brought to you for free and open access by the National Aeronautics and Space Administration at DigitalCommons@University of Nebraska - Lincoln. It has been accepted for inclusion in NASA Publications by an authorized administrator of DigitalCommons@University of Nebraska - Lincoln. 


\title{
Non-Linear Filtering and Limiting in High Order Methods for Ideal and Non-Ideal MHD
}

\author{
H. C. Yee ${ }^{1}$ and B. Sjögreen ${ }^{2}$
}

Received October 22, 2004; accepted (in revised form) January 28, 2005; Published online January 5, 2006

\begin{abstract}
The adaptive nonlinear filtering and limiting in spatially high order schemes (Yee et al. J. Comput. Phys. 150, 199-238, (1999), Sjögreen and Yee, J. Scient. Comput. 20, 211-255, (2004)) for the compressible Euler and Navier-Stokes equations have been recently extended to the ideal and non-ideal magnetohydrodynamics (MHD) equations, (Sjögreen and Yee, (2003), Proceedings of the 16th AIAA/CFD conference, June 23-26, Orlando F1; Yee and Sjögreen (2003), Proceedings of the International Conference on High Performance Scientific Computing, March, 10-14, Honai, Vietnam; Yee and Sjögreen (2003), RIACS Technical Report TR03. 10, July, NASA Ames Research Center; Yee and Sjögreen (2004), Proceedings of the ICCF03, July 12-16, Toronto, Canada). The numerical dissipation control in these adaptive filter schemes consists of automatic detection of different flow features as distinct sensors to signal the appropriate type and amount of numerical dissipation/filter where needed and leave the rest of the region free from numerical dissipation contamination. The numerical dissipation considered consists of high order linear dissipation for the suppression of high frequency oscillation and the nonlinear dissipative portion of high-resolution shock-capturing methods for discontinuity capturing. The applicable nonlinear dissipative portion of high-resolution shock-capturing methods is very general. The objective of this paper is to investigate the performance of three commonly used types of discontinuity capturing nonlinear numerical dissipation for both the ideal and non-ideal MHD.
\end{abstract}

KEY WORDS: High order schemes; filtering; nonlinear filtering; limiting; MHD; shock-capturing; shock/turbulence interactions.

${ }^{1}$ NASA Ames Research Center, Moffett Field, Calif., 94035, USA.

2 The Royal Institute of Technology, 10044 Stockholm, Sweden. 


\section{SOLVING THE CONSERVATIVE SYSTEM USING THE SYMMETRIZABLE EIGENVECTORS}

Consider the 3-D conservative and symmetrizable $[6,12]$ (non-conservative) forms of the ideal compressible MHD equations in Cartesian grids,

$$
\begin{array}{cc}
U_{t}+\nabla \cdot \mathbf{F}=0 & \text { (conservative) } \\
U_{t}+\nabla \cdot \mathbf{F}=S & (\text { symmetrizable }),
\end{array}
$$

$$
U=\left(\begin{array}{c}
\rho \\
\rho u \\
\rho v \\
\rho w \\
e \\
B_{x} \\
B_{y} \\
B_{z}
\end{array}\right)_{t}{ }_{t} \mathbf{\mathbf { u }}=\left(\begin{array}{c}
\rho \mathbf{u u}^{T}+\left(p+B^{2} / 2\right) I-\mathbf{B B}^{T} \\
\mathbf{u}\left(e+p+B^{2} / 2\right)-\mathbf{B}\left(\mathbf{u}^{T} \mathbf{B}\right) \\
\mathbf{u B}^{T}-\mathbf{B u}^{T}
\end{array}\right) ; \quad S=-(\nabla \cdot \mathbf{B})\left(\begin{array}{c}
0 \\
B_{x} \\
B_{y} \\
B_{z} \\
\mathbf{u}^{T} \mathbf{B} \\
u \\
v \\
w
\end{array}\right) .
$$

Here the velocity vector $\mathbf{u}=(u, v, w)^{\mathrm{T}}$, the magnetic field vector $\mathbf{B}=$ $\left(B_{x}, B_{y}, B_{z}\right)^{T}, \rho$ is the density, and $e$ is the total energy. The notation $B^{2}=$ $B_{x}^{2}+B_{y}^{2}+B_{z}^{2}$ is used. The pressure is related to the other variables by

$$
p=(\gamma-1)\left(e-\frac{1}{2} \rho\left(u^{2}+v^{2}+w^{2}\right)\right)-\frac{1}{2}\left(B_{x}^{2}+B_{y}^{2}+B_{z}^{2}\right) .
$$

For plasmas and monatomic gases, $\gamma=5 / 3$. The vector on the right hand side of (1.2) is the non-conservative portion of the symmetrizable MHD equations and is frequently referred to in the literature as a source term vector. The conservative and symmetrizable forms of the non-ideal compressible MHD [5] take the form

$$
\begin{gathered}
U_{t}+\nabla \cdot \mathbf{F}=\mathbf{F}_{v}, \\
U_{t}+\nabla \cdot \mathbf{F}=\mathbf{F}_{v}+S, \\
\mathbf{F}_{v}=\left[0 \operatorname{div} \tau \operatorname{div}\left(\mathbf{u}^{T} \tau\right)+\operatorname{divh}-\frac{1}{\sigma} \operatorname{div}((\nabla \times \mathbf{B}) \times \mathbf{B}) \frac{1}{\sigma}(\triangle \mathbf{B}-\nabla \operatorname{div} \mathbf{B})\right] .
\end{gathered}
$$

The vector $\mathbf{F}_{v}$ includes viscosity, resistivity, and conductivity with $\tau$ being the viscous stress tensor, $\sigma$ the conductivity coefficient, and $\mathbf{h}$ the heat flux.

For non-ideal MHD, we apply the inviscid MHD base scheme twice for the viscous flux derivatives (similarly for the resistive terms). There is no viscous filtering involved. Without lost of generality, we will describe 
our numerical methods for the inviscid $x$-flux of the ideal MHD (1.1) on a uniform grid. The schemes to be discussed, in most part, only spell out the $x$-component terms with the $y$-and $z$-components omitted. Let $A(U)$ denote the Jacobian $\partial F / \partial U$ with the understanding that the present $F$ and $S$ are the inviscid $x$-component of the 3-D description above. We also write the non-conservative term $S$, in the $x$-direction as $N(U) U_{x}$.

An important ingredient in our high order filter method is the use of the dissipative portion of high-resolution shock-capturing schemes as part of the nonlinear filters for discontinuity capturing. If the dissipative portion of higher order Lax-Friedrichs or Nessyahu-Tadmor [9] type of shock-capturing schemes are not employed (see [17] for a discussion), these nonlinear filters usually involve the use of approximate Riemann solvers.

Seven of the eigenvalues and eigenvectors are identical for the "conservative" Jacobian matrix $A$ and the "symmetrizable" Jacobian matrix $(A-N)$ [4]. For ease of reference, we refer to the distinct eigenvalue (eigenvector) between the conservative and symmetrizable MHD as the eighth eigenvalue (eigenvector). The eighth eigenvector of $A$ of the conservative system associated with the degenerate zero eigenvalue can sometimes coincide with one of the other eigenvectors, thereby, making it difficult to obtain the Roetype approximate Riemann solver for the multi-dimensional conservative MHD. On the other hand, the eigenvectors of the symmetrizable Jacobian $A^{*}=(A-N)$ always form a complete basis, and can be obtained from analytical formulas $[6,12]$ for 1-D or higher. Here, a Roe-type average state developed in Gallice [4] for the multi-D symmetrizable MHD is employed. This form is an improvement over the Brio \& Wu [1] and Powell [12] forms.

For strong shocks, to ensure the correct shock strength and location, we prefer to solve the conservative MHD system instead of the symmetrizable MHD. As in [15, 20, 21], we use eigenvectors of the symmetrizable form but with the degenerate eigenvalue replaced by an entropy correction [7, 17] (a small parameter $\epsilon$ that is scaled by the largest eigenvalue of $A(U)$ ) for the conservative form. For more than one-space dimension, a multi-dimension entropy correction [17] is used for each of the degenerate eigenvalues in each spatial direction. Our rationale for doing this is that the incorrect eigenvector for the conservative form will be multiplied by an eigenvalue which is close to zero. Thus the effect of a "false" eigenvector will be small. Note that in the present context, the use of the an entropy correction is different from the standard entropy correction associated with expansion shocks in the Roe-type approximate solver in gas dynamics since the conservative inviscid gas dynamics equations are strictly hyperbolic. 


\section{DESCRIPTION OF HIGH ORDER FILTER METHODS}

Our filter idea is very general and can be used in conjunction with spectral, compact and non-compact spatially central base schemes. Basically, the filter method consists of two steps, a divergence-free preserving (base scheme) step (not involving the use of approximate Riemann solvers or flux limiters) and a filter step (usually involving the use of approximate Riemann solvers and flux limiters). In order to have good shock-capturing capability and improved nonlinear stability related to spurious high frequency oscillations, the blending of a high order nonlinear filter and a high order linear filter was proposed in $[19,21,23]$. The nonlinear filter consists of the product of an artificial compression method (ACM) indicator or wavelet sensor and the nonlinear dissipative portion of a high-resolution shock-capturing scheme. The high order linear filter consists of the product of another sensor, a tuning parameter and a high order centered linear dissipative operator that is compatible with the order of the base scheme being used. Due to space limitation, only the nonlinear filter using wavelets as sensors is presented here. The numerical examples considered solve the conservative system and exhibit highly accurate solutions without the use of high order linear filters.

\subsection{Divergence-Free Preserving Base Scheme Step}

The first step of the numerical method consists of a time step via a high order non-dissipative spatial and high order temporal base scheme operator $L^{*}$. After the completion of a full time step of the base scheme step, the solution is denoted by $U^{*}$

$$
U^{*}=L^{*}\left(U^{n}\right),
$$

where $U^{n}$ is the numerical solution vector at time level $n$. For strong shock interactions, high order linear dissipation is added to the base scheme step. For example, an eighth-order linear dissipation with the sixth-order centered base scheme to approximate $F(U)_{x}$ (with the grid indices $k$ and $l$ for the $y$ - and $z$-directions suppressed) is written as

$$
\frac{\partial F}{\partial x} \approx D_{06} F_{j}+d(\Delta x)^{7}\left(D_{+} D_{-}\right)^{4} U_{j}
$$

where $D_{06}$ is the standard sixth-order accurate centered difference operator, and $D_{+} D_{-}$is the standard second-order accurate centered approximation of the second derivative. The small parameter $d$ is a scaled value in the range of 0.00001 to 0.001 , depending on the flow problem, and has the sign which gives dissipation in the forward time direction. The $D_{06}$ operator 
is modified at boundaries in a stable way by the so called summation-bypart (SBP) operators $[10,11,19]$. The linear numerical dissipation operator $D_{+} D_{-}$is modified at the boundaries to be semi-bounded [4]. This highly accurate spatial base scheme is employed to numerically preserve the divergence-free condition of the magnetic field (to the level of round-off error) for uniform Cartesian grids with periodic boundary conditions, see [23] for the comparison of compact and non-compact central differencing as base schemes.

\subsection{Adaptive Numerical Dissipation Filter Step}

After the completion of a full time step of the divergence-free preserving base scheme step, the second step is to adaptively filter the solution by the product of a "wavelet sensor" and the "nonlinear dissipative portion of a high-resolution shock-capturing scheme" (involving the use of flux limiters). If necessary, the blending of a high order linear filter with a nonlinear filter [19] can be employed but will not be discussed here. See [23] for a multistep filter approach. The final update step after the filter step can be written (with some of grid indices suppressed for ease of illustration) as

$$
\begin{aligned}
U_{j, k, l}^{n+1}= & U_{j, k, l}^{*}-\frac{\Delta t}{\Delta x}\left[H_{j+1 / 2}^{f x}-H_{j-1 / 2}^{f x}\right]-\frac{\Delta t}{\Delta y}\left[H_{k+1 / 2}^{f y}-H_{k-1 / 2}^{f y}\right] \\
& -\frac{\Delta t}{\Delta z}\left[H_{l+1 / 2}^{f z}-H_{l-1 / 2}^{f z}\right] .
\end{aligned}
$$

Here, $H_{j \pm 1 / 2}^{f x}, H_{k \pm 1 / 2}^{f y}$ and $H_{l \pm 1 / 2}^{f z}$ are the filter numerical fluxes in the $x, y$ and $z$-directions, respectively. The $x$-filter numerical flux vector $H_{j+1 / 2}^{f x}$ is

$$
H_{j+1 / 2}^{f x}=R_{j+1 / 2} \bar{H}_{j+1 / 2},
$$

where $R_{j+1 / 2}$ is the matrix of right eigenvectors of the Jacobian of the non-conservative MHD flux vector $\left(A_{j+1 / 2}-N_{j+1 / 2}\right)$ evaluated at the Gallice average state [4] in terms of the $U^{*}$ solution from the base scheme step (2.4). The subscript in $R_{j+1 / 2}$ indicates the average state evaluated in the $x$-direction of the eigenvectors in terms of $U^{*}$. See [4] or Appendix A of [21] for the average state formula for the 3-D non-conservative system (1.2). The $\bar{H}_{j+1 / 2}$ (involving the use of wavelet sensors and flux limiters) are also evaluated from the same average state. The dimension-by-dimension procedure of applying the approximate Riemann solver is adopted. 
Denote the elements of the vector $\bar{H}_{j+1 / 2}$ by $\bar{h}_{j+1 / 2}^{l}, l=1,2, \ldots, 8$. The nonlinear portion of the filter $\bar{h}_{j+1 / 2}^{l}, l=1,2, \ldots, 8$, has the form

$$
\bar{h}_{j+1 / 2}^{l}=\frac{1}{2}\left(s^{N}\right)_{j+1 / 2}^{l}\left(\phi_{j+1 / 2}^{l}\right)
$$

Here $\left(s^{N}\right)_{j+1 / 2}^{l}$ is the sensor to activate the higher order nonlinear numerical dissipation $\phi_{j+1 / 2}^{l}$. For example, $\left(s^{N}\right)_{j+1 / 2}^{l}$ is designed to be zero or near zero in regions of smooth flow and near one in regions with discontinuities. $\left(s^{N}\right)_{j+1 / 2}^{l}$ varies from one grid point to another and is obtained from a wavelet analysis of the flow solution [14]. The wavelet sensor can be obtained from the characteristic variables for each wave or a single sensor for all eight waves, based on pressure and density. Both methods were implemented but for the numerical tests in this paper, the simpler non-characteristic sensor was employed.

The dissipative portion of the nonlinear filter $\Phi_{j+1 / 2}^{l}=g_{j+1 / 2}^{l}-b_{j+1 / 2}^{l}$ is the dissipative portion of a high order high-resolution shock-capturing scheme for the local $l$ th-characteristic wave. Here $g_{j+1 / 2}^{l}$ and $b_{j+1 / 2}^{l}$ are numerical fluxes of the uniformly high order high-resolution scheme and a high order central scheme for the $l$ th characteristic, respectively. It is noted that $b_{j+1 / 2}^{l}$ might not be unique since there is more than one way of obtaining $\phi_{j+1 / 2}^{l}$.

Three forms of nonlinear dissipation $\phi_{j+1 / 2}^{l}$ are considered, namely:

- Dissipative portion of the fifth-order WENO scheme (WENO5) [8]. It can be obtained e.g., in the $x$-direction by taking the full WENO5 scheme in the $x$-direction and subtracting $D_{06} F_{j}$.

- Dissipative portion of the a second-order MUSCL scheme [18].

- Dissipative portion of the Harten-Yee TVD scheme [18, 21].

The nonlinear filter given by (2.7), if applied to the entire MHD system, will not preserve the divergence free magnetic field condition in general. For the computations in this paper, the "No filter on B" option is chosen. That is, the nonlinear filter step (2.7) only applies to the first five equations of (1.1) or (1.2). Here the complete set of eigenvalues and eigenvectors of the full symmetrizable MHD system is used to evaluate the first five equations of (1.1) or (1.2). With the divergence free spatial base scheme, the divergence free property should be preserved. Extensive grid convergence comparison of the "no filter on B" with the "filter all of the MHD equations" (filter all) options were presented in [21]. Alterna- 
tive approach in obtaining divergence-free preserving shock-capturing filters follow in a similar vein as the constrained transport approach [3].

\section{2-D COMPRESSIBLE IDEAL AND NON-IDEAL MHD NUMERICAL EXAMPLES}

The wavelet filter schemes using the dissipative portion of WENO5, second-order MUSCL and Harten-Yee TVD schemes with sixth-order spatial central base scheme $(d=0$ in (2.5)) for both the inviscid and viscous MHD flux derivatives and a fourth-order Runge-Kutta method are denoted by WAV66weno5, WAV66mus and WAV66hy respectively. The first number indicates the order of the base scheme for discretizing the inviscid flux derivatives. The second number indicates the order of the scheme for discretizing the viscous flux derivatives, if present. To adhere to the convention of previous work, even though when dealing with inviscid flows, the same notation is used. Viscous flows are indicated with a non-zero Reynolds number. As mentioned before, there is no filtering for the viscous fluxes. If an eighthorder linear dissipation $(d \neq 0$ in (2.5)) is used for the base scheme, the symbol "AD8" is added as in WAV66weno5+AD8. Computation using the same temporal and spatial scheme for the viscous MHD flux derivatives, and the standard fifth-order WENO scheme for the inviscid flux derivatives is denoted by WENO5. Computations using a second-order MUSCL and the Harten-Yee [21] TVD scheme for the inviscid MHD flux with the secondorder central scheme for the viscous flux and a second-order Runge-Kutta method are denoted by MUSCL and HY, respectively.

The entropy fix parameter $\epsilon$ is 0.25 [7, 17] for the Harten-Yee, MUSCL, WAV66mus and WAV66hy schemes (to avoid expansion shocks and carbuncle phenomenon). The same value of $\epsilon$ is used for the degenerate zero eigenvalue of the conservative system. The cut off wavelet Lipschitz exponent $\beta$ is 0.5 [14] for all the wavelet filter schemes. See [14, $18,19]$ or Appendix B of [21] for the definition of $\epsilon$ and $\beta$. Except for WENO5, the van Leer version of the van Albada limiter is used. For the second-order MUSCL scheme, the limiter is applied to the primitive variables. For viscous computations, the Reynolds number $R e=1000$, resistivity coefficient of 100 and a Prantdl number of 0.72 are used for all test cases. Extensive grid convergence studies using WAV66hy and ACM66hy (using ACM instead of wavelet as the sensor) for typical ideal and nonideal MHD test cases were conducted in [15, 21, 22]. More accurate solutions were obtained with WAV66hy and ACM66hy than with WENO5, which is more CPU intensive. The following investigates the performance of the three different filters solving the conservative MHD. 


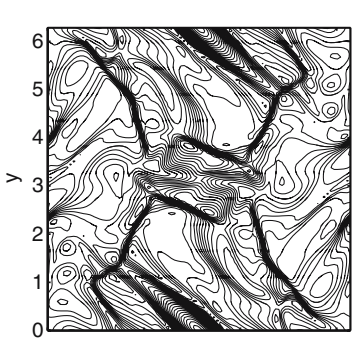

WAV66weno5, 101×101

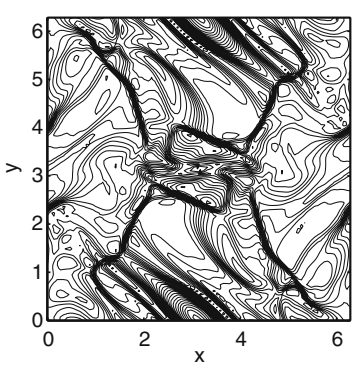

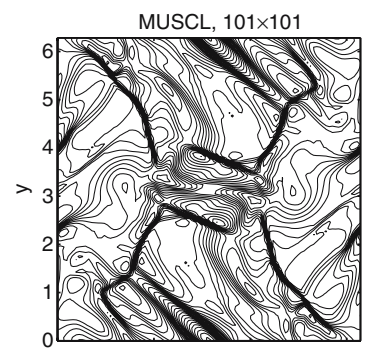

WAV66mus, $101 \times 101$

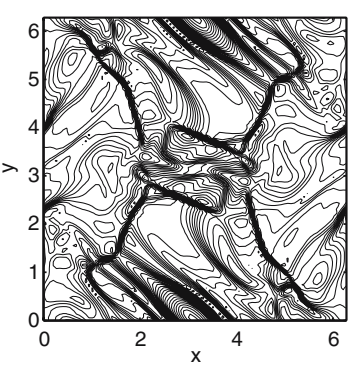

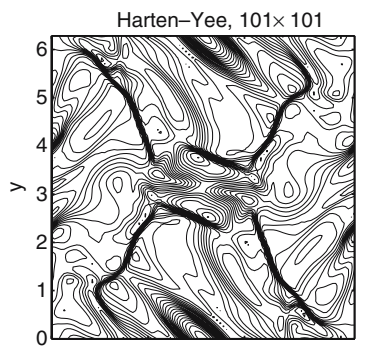

WAV66, $101 \times 101$

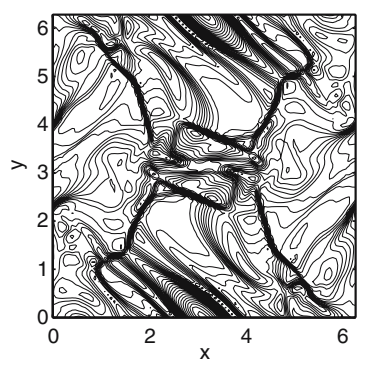

Fig. 1. Inviscid Orszag-Tang Problem using a $101 \times 101$ grid. Density contours for the ideal MHD at $T=3.14$. Top row: WENO5(left), MUSCL(middle), HY(right). Bottom row: WAV66weno5(left), WAV66mus(middle), WAV66hy(right).

\subsection{Compressible Orszag-Tang Vortex $(\gamma=5 / 3$, Periodic BC)}

The 2-D compressible Orszag-Tang vortex problem [2] consists of periodic boundary conditions with smooth initial data.

$\left(\rho, u, v, w, p, B_{x}, B_{y}, B_{z}\right)=(25 / 9,-\sin y, \sin x, 0,5 / 3,-\sin y, \sin 2 x, 0)$.

The computational domain is $0<x<2 \pi, 0<y<2 \pi$ and the computation stops at time $T=3 \cdot 14(\approx \pi)$, when complicated structure and discontinuities have formed and interacted. Density contours with 30 equally spaced contours between 0.9 and 6.1 are used for illustration. Figures 1 and 2 show the comparison among the three filter schemes (no filter on $\mathbf{B}$ option), WENO5, MUSCL and Harten-Yee (HY) using uniform $101 \times 101$ and $801 \times 801$ grids for the inviscid MHD. Figures 3 and 4 show the corresponding viscous MHD comparison.

Grid convergence was obtained by all six methods using the $801 \times 801$ grid. Computations based on a $1601 \times 1601$ grid are used as the reference solutions. For $51 \times 51-401 \times 401$ grids, small structures are better captured by the three filter methods than WENO5, MUSCL or Harten-Yee (see e.g., $2<x<4,0<y<1$ ). In addition, for the inviscid case, the three filter methods are more stable than the other three methods in the sense larger CFL 

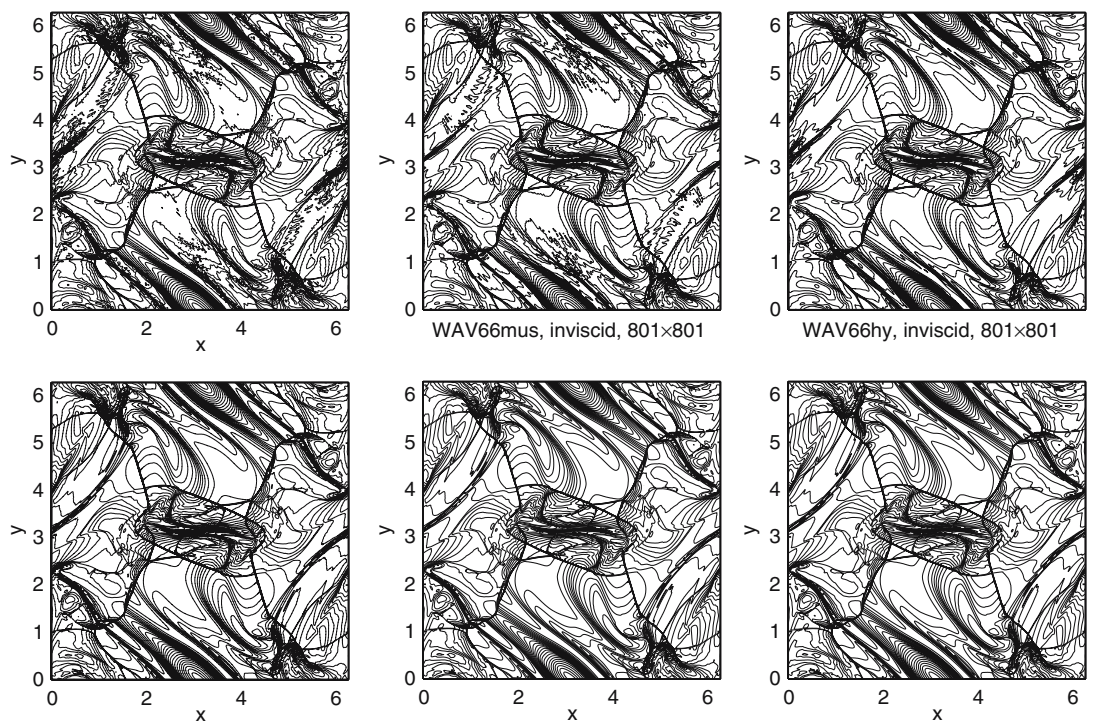

Fig. 2. Inviscid Orszag-Tang Problem using a $801 \times 801$ grid. Density contours for the ideal MHD at $T=3.14$. Top row: WENO5(left), MUSCL(middle), HY(right). Bottom row: WAV66weno5(left), WAV66mus(middle), WAV66hy(right).

number can be used. Figure 2 shows the computations using a CFL of 0.6 and an $801 \times 801$ grid. WENO5 and MUSCL show a slight small oscillation. These oscillations can be suppressed by applying the limiter in the characteristic variables in the MUSCL scheme (figures not shown).

For the viscous case, the flow structure is less complicated than that of the inviscid case. All computations use a CFL of 0.6. For coarse grids, again small structures are better captured by the three filter methods than by WENO5, MUSCL or Harten-Yee. In other words, the three filter methods exhibit similar accuracy as the three standard shock-capturings methods with a coarse grid. For both the inviscid and viscous computations, all three filter methods using the "no filter on B" option are divergence-free preserving, whereas the "filter all" option as well as the standard WENO5, MUSCL and HY without divergence cleaning are not divergence free. Their $\nabla \cdot \mathbf{B}$ numerical error at $T=3.14$ increases as the grid is refined. See [21] for some illustrations.

In general, MUSCL and Harten-Yee require similar CPU time. The CPU required by the three filter methods is within $15 \%$ depending on the problem, grid spacings and time steps. They require slightly more CPU time $(20 \%)$ than the Harten-Yee and MUSCL schemes. This is due to the fact that all filter schemes require only one Riemann solve per time step 

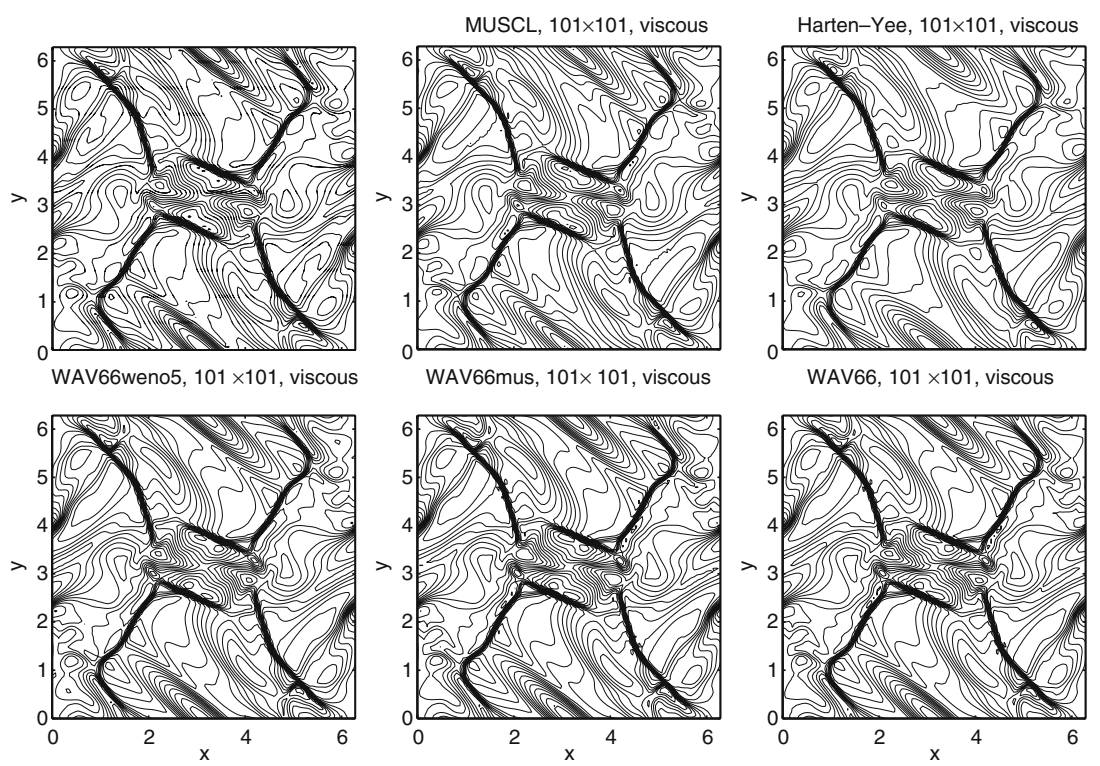

Fig. 3. Viscous Orszag-Tang Problem using a $101 \times 101$ grid. Density contours for non-ideal MHD with $R e=1000$, and resistivity coeff. of 100 at $T=3.14$. Top row: WENO5(left), MUSCL(middle), HY(right). Bottom row: WAV66weno5(left), WAV66mus(middle), WAV66hy(right).

per direction (independent of the time discretizations of the base scheme step) as opposed to two Riemann solves per time step per direction by the MUSCL and Harten-Yee schemes using a second-order Runge-Kutta method. WENO5 requires at least twice the CPU time of all other methods since four Riemann solves per time step per direction are required by WENO5.

\subsection{A Planar Shock Interacting with a Magnetic Cloud $(\gamma=5 / 3$, Supersonic Inflow \& Open Boundaries)}

The second test problem is a planar shock interacting with a magnetic cloud studied in [2]. This is a more challenging problem to simulate due to the stiffness of the flow with rapidly developing complex wave interactions close to the boundaries. The same initial configuration as in [16] is considered here. The computational domain is the square $0<x<1,0<y<1$. A planar shock is initially situated at $x=0.6$ and moves towards the right.

$$
\begin{gathered}
\left(\rho, u, v, w, p, B_{x}, B_{y}, B_{z}\right)_{L}=(3.86859,0,0,0,167.345,0,2.1826182,-2.1826182), \\
\left(\rho, u, v, w, p, B_{x}, B_{y}, B_{z}\right)_{R}=(1,-11.2536,0,0,1,0,0.56418958,-0.56418958) .
\end{gathered}
$$



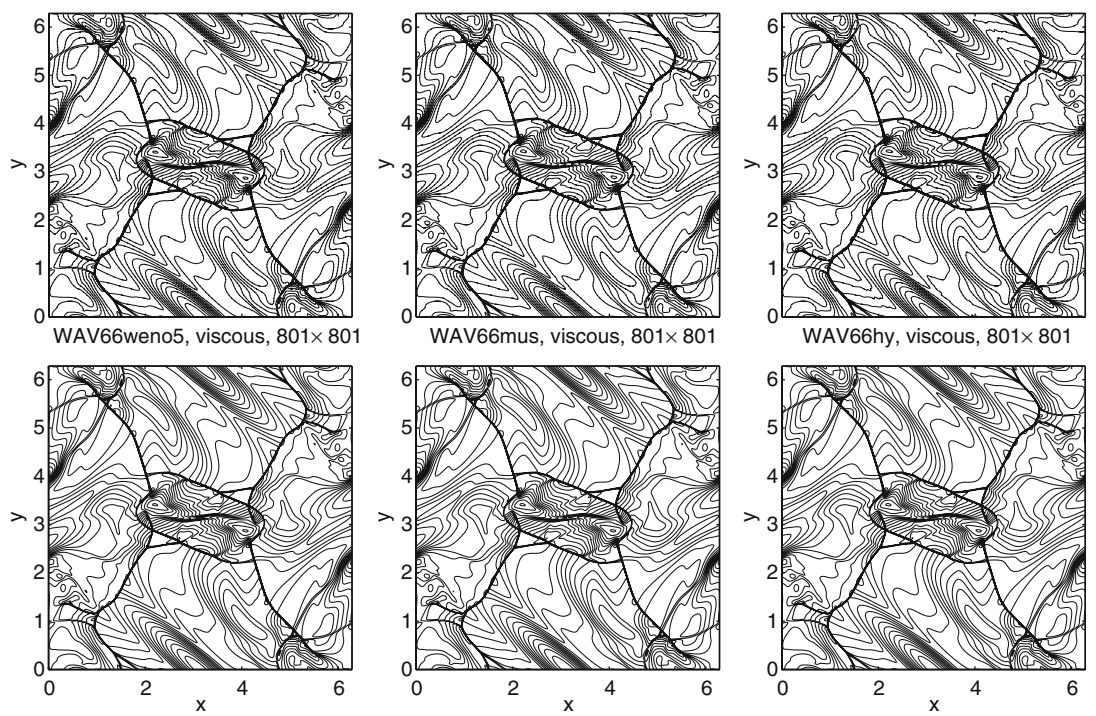

Fig. 4. Viscous Orszag-Tang Problem using a $801 \times 801$ grid. Density contours for non-ideal MHD with $R e=1000$, and resistivity coeff. of 100 at $T=3.14$. Top row: WENO5(left), MUSCL(middle), HY(right). Bottom row: WAV66weno5(left), WAV66mus(middle), WAV66hy(right).

Inside the cloud with center at $(0.8,0.5)$ and radius 0.15 , a state of increased density is given by the initial state:

$\left(\rho, u, v, w, p, B_{x}, B_{y}, B_{z}\right)_{C}=(10,-11.2536,0,0,1,0,0.56418958,-0.56418958)$.

The flow velocity is directed in the negative $x$-direction, and the cloud will move to the left. The right boundary is supersonic inflow, where the right state is imposed. The other boundaries are open boundaries. Density contours with 50 equidistant contours in $\log$ scale from $\log (0.99)$ to $\log (48)$ are used.

Figures 5 and 6 show the comparison among the three filter schemes (WAV66weno5+AD8, WAV66mus+AD8, and WAV66hy+AD8, with $d=0.001$ in (2.5)), with the "no filter on B option", WENO5, MUSCL and Harten-Yee using uniform $201 \times 201$ and $801 \times 801$ grids for the inviscid MHD. Computations using WAV66weno5, WAV66mus, and WAV66hy $(d=0$ in (2.5)) are not as stable. For the viscous case, AD8 (i.e., $d \neq 0$ ) is not necessary as part of the base scheme for a stable solution. Due to limited space, these figures are not shown.

As opposed to the Orszag-Tang problem, in this case, grid convergence was not quite achieved for all six methods using the $801 \times 801$ grid for the 

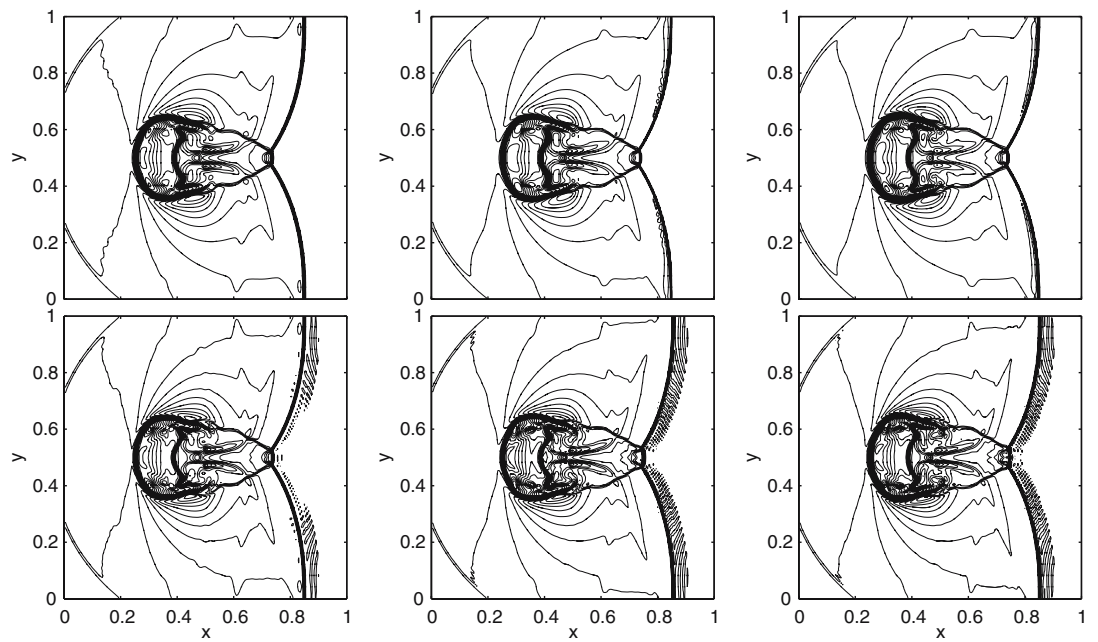

Fig. 5. Inviscid Magnetic Cloud Problem using a $201 \times 201$ grid. Density contours for the ideal MHD at $T=0.06$. Top row: WENO5(left), MUSCL(middle), HY(right). Bottom row: WAV66weno5+AD8(left), WAV66mus+AD8(middle), WAV66hy+AD8(right).
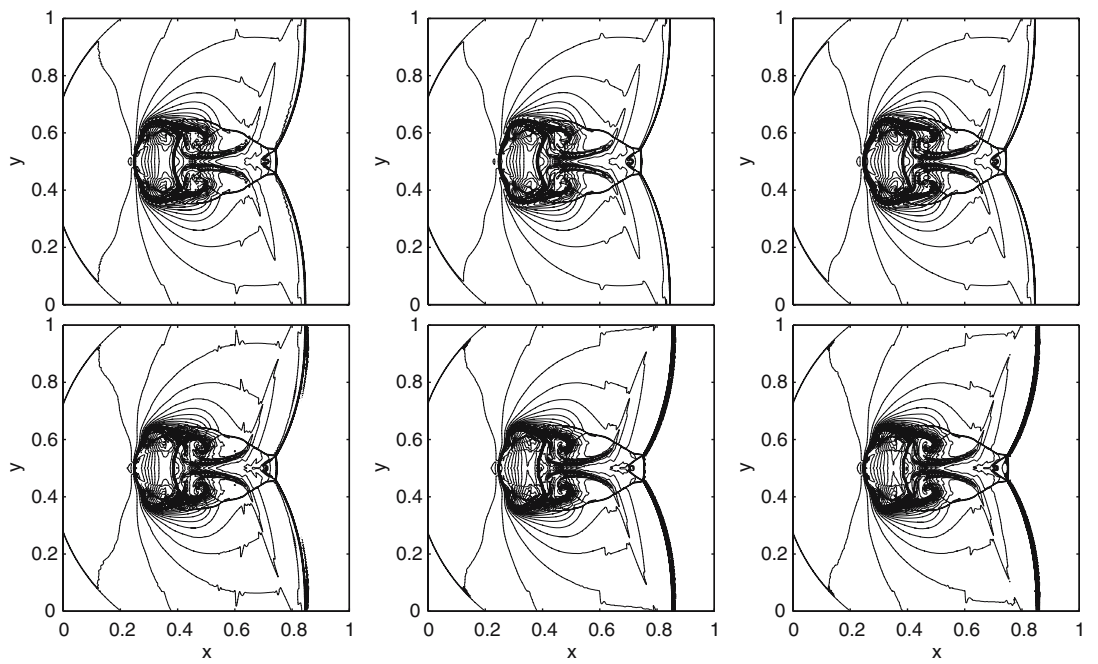

Fig. 6. Inviscid Magnetic Cloud Problem using a $801 \times 801$ grid. Density contours for the ideal MHD at $T=0.06$. Top row: WENO5(left), MUSCL(middle), HY(right). Bottom row: WAV66weno5+AD8(left), WAV66mus+AD8(middle), WAV66hy+AD8(right). 

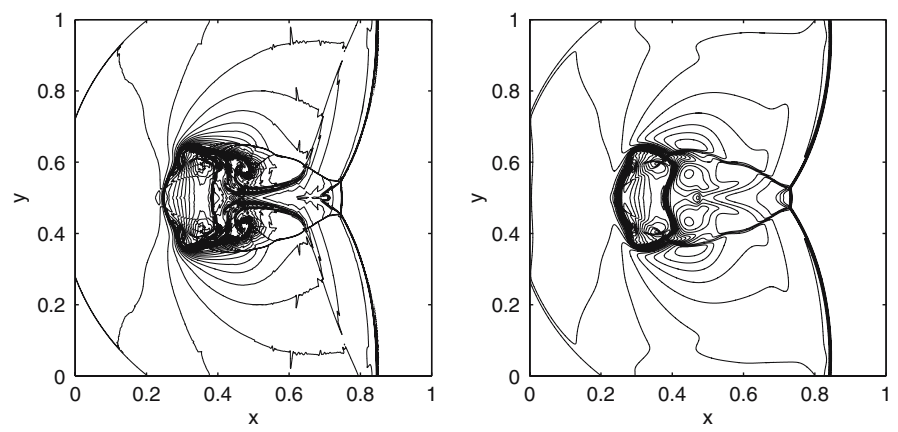

Fig. 7. Inviscid and Viscous Magnetic Cloud Problem using a $1601 \times 1601$ grid by WAV66weno5+AD8 $(\mathrm{d}=0.001)$. Density contours for ideal and non-ideal MHD with $R e=1000$, and resistivity coeff. of 100 at $T=0.06$, inviscid (left) and viscous (right).

inviscid case. For both the viscous and inviscid cases, overall, the three filter methods are more accurate than the other three methods. Computations based on a $1601 \times 1601$ grid are used as the reference solutions (see Fig. 7). For $51 \times 51-101 \times 101$ grids, small structures are better captured by the three filter methods than by WENO5, MUSCL and Harten-Yee (e.g., inside the cloud region). Moreover, among the three filter methods, WAV66hy+AD8 is slightly more accurate on capturing the fine scale structures in most coarse grid cases. It is interesting to see the contrast in the flow structure between the inviscid and viscous converged solutions (Fig. 7).

For all three filter methods using the "no filter on B" option, perfect $\nabla \cdot \mathbf{B}$ preservation within machine zero (figures not shown) is only obtained up to time $T=0.04$. The increase in the norm of $\nabla \cdot \mathbf{B}$ is caused by boundary effects (see [21] for some illustrations). A SBP difference boundary operator is used $[10,11]$. Due to the wide grid stencil of the SBP boundary difference operator in conjunction with the need to use an extrapolation to the outermost open boundary point, $\nabla \cdot \mathbf{B}$ is not preserved. The effect is only seen when the solution is non-trivial on the boundary where complex wave interactions are taking place in both directions of the open boundaries. Although divergence-free preservation by the three filter methods is not possible for $T>0.04$ by the "no filter on B" option, the $L^{2}$-norm of $\nabla \cdot \mathbf{B}$ for this option is at least an order of magnitude smaller than the "filter all" option and the three standard shock-capturing schemes when $T>0.04$. See $[20,21]$ for some illustrations of WAV66hy+AD8. An alternative in obtaining a divergence-free solution is to employ standard divergence free cleaning on the filter step. This is a subject of on going research. The relative CPU required by the six methods is the same as the previous test case. 


\section{CONCLUDING REMARKS}

The performance of three nonlinear filters for the adaptive numerical dissipation control in high order methods $[14,15,18,21]$ is investigated. The "no filter on B" option by the three filter methods works well for both conservative and symmetrizable systems (computations not shown) and exhibits smaller $\nabla \cdot \mathbf{B}$ numerical error than standard shock-capturing methods without traditional divergence cleanings. For periodic boundary conditions and for open boundaries without complex wave interactions near the physical boundaries, these filter schemes are divergence free. In general, for coarse grids, the high order methods are more accurate and require less grid points than required by second-order methods. For fine enough grids, in most test cases, the accuracy is similar for all six methods.

\section{REFERENCES}

1. Brio, M., and Wu, C. C. (1988). An upwind differencing scheme for the equations of ideal magnetohydrodynamics. J. Comput. Phys. 75, 400-422.

2. Dai, W., and Woodward, P. R. (1998). A simple finite difference scheme for multidimensional magnetohydrodynamical equations. J. Comput. Phys. 142, 331-369.

3. Evans, C. R., and Hawley, J. F. (1988). Simulation of magnetohydrodynamic flows: a constrained transport method, Astrophys. J. 332, 659-677.

4. Gallice, G. (1997). Systéme D'Euler-Poisson, Magnétohydrodynamique et Schemeas de Roe, $\mathrm{PhD}$ Thesis, L'Université Bordeaux I.

5. Gaitonde, D. V. (1999). Development of a Solver for 3-D Non-Ideal Magnetogasdynamics AIAA Paper 99-3610.

6. Godunov, S. K. (1972). Symmetric form of the equations of magnetohydrodynamics, numerical methods for mechanics of continuum medium. 13(1), 26-34.

7. Harten, A., and Hyman, J. M. (1983). A self-adjusting grid for the computation of weak solutions of hyperbolic conservation laws. J. Comput. Phys. 50, 235-269.

8. Jiang, G.-S., and Shu, C.-W. (1996). Efficient implementation of weighted ENO schemes. J. Comput. Phys. 126, 202-228.

9. Nessyahu, H., and Tadmor, E. (1990). Non-oscillatory central differencing for hyperbolic conservation laws. J. Comput. Phys. 87, 408-463.

10. Nordstrom, J., and Carpenter, M. H. (1999). Boundary and interface conditions for highorder finite-difference schemes applied to the Euler and Navier-Stokes equations. J. Comput. Phys. 148, 621-645.

11. Olsson, P. (1995). Summation by parts, projections and stability. I. Math. Comp. 64, 1035-1065.

12. Powell, K. G. (1994). An Approximate Riemann Solver for Magnetohydrodynamics (That works in More than One Dimension), ICASE-Report 94-24, NASA Langley Research Center, April.

13. Sjögreen, B. (1995). High order centered difference methods for the compressible NavierStokes equations. J. Comput. Phys. 117, 67-78.

14. Sjögreen, B., and Yee, H. C. (2004). Multiresolution wavelet based adaptive numerical dissipation control for shock-turbulence computation, RIACS Technical Report TR01.01, NASA Ames research center (Oct 2000); also, J. Scient. Comput. 20, 211-255. 
15. Sjögreen, B., and Yee, H. C. (2003). Efficient Low Dissipative High Order Schemes for Multiscale MHD Flows, I: Basic Theory, AIAA 2003-4118, Proceedings of the 16th AIAA/CFD Conference, June 23-26, Orlando, Fl.

16. Tóth, G. (2000). The div $B=0$ constraint in shock-capturing magnetohydrodynamic codes. J. Comput. Phys. 161, 605-652.

17. Yee, H. C. (1989). A Class of High-Resolution Explicit and Implicit Shock-Capturing Methods, VKI Lecture Series 1989-04, March 6-10, also NASA TM-101088, Feb. 1989.

18. Yee, H. C., Sandham, N. D., and Djomehri, M. J. (1999). Low dissipative high order shockcapturing methods using characteristic-based filters. J. Comput. Phys. 150, 199-238.

19. Yee, H. C., and Sjögreen, B. (2002). Designing Adaptive Low Dissipative High Order Schemes for Long-Time Integrations, Turbulent Flow Computation, (Eds. D. Drikakis \& B. Geurts), Kluwer Academic Publisher; also RIACS Technical Report TR01-28, Dec. 2002.

20. Yee, H. C., and Sjögreen, B. (2003). Divergence Free High Order Filter Methods for the Compressible MHD Equations, Proceedings of the International Conference on High Performance Scientific Computing, March 10-14, 2003, Hanoi, Vietnam.

21. Yee, H. C., and Sjögreen, B. (2003). Efficient Low Dissipative High Order Scheme for Multiscale MHD Flows, II: Minimization of Div(B) Numerical Error, RIACS Technical Report TR03.10, July NASA Ames Research Center.

22. Yee, H. C., and Sjögreen, B. (2004). Adaptive Numerical Dissipation Control in High Order Schemes for 3-D Non-Ideal MHD, Proceedings of the ICCFD3, July 12-16, Toronto, Canada.

23. Yee, H. C., and Sjögreen, B. (2005). Nonlinear Filtering in Compact High Order Schemes, Proceedings of the ICNSP, July 12-15, Nara, Japan. 